\title{
Scheme for Secure Communication via Information Hiding Based on Key Exchange and Decomposition Protocols
}

\author{
Boris S. Verkhovsky \\ Computer Science Department, New Jersey Institute of Technology, University Heights, Newark, USA \\ E-mail:verb@njit.edu \\ Received January 26, 2011; revised February 8, 2011; accepted February 9, 2011
}

\begin{abstract}
This paper considers a decomposition framework as a mechanism for information hiding for secure communication via open network channels. Two varieties of this framework are provided: one is based on Gaussian arithmetic with complex modulus and another on an elliptic curve modular equation. The proposed algorithm is illustrated in a numerical example.
\end{abstract}

Keywords: Complex Modulus, Cryptanalytic Protection, Decomposition, Gaussian Modular Arithmetic, Information Hiding, Key Exchange, Modular Elliptic Curve, Secure Communication

\section{Introduction and Problem Definition}

In this paper it is demonstrated how to use various Diffie-Hellman key establishment (DHKE) protocols in order to design a computationally efficient cryptographic schemes for secure communication between two parties \{called Alice and Bob\}. One of these key establishment protocols is based on modular elliptic curves (ECDHKE) [1]. Another DHKE protocol is based on arithmetic of complex integers with complex modulus [2].

DHKE protocol based on complex integers: In this scheme both parties agree to select a Gaussian integer $L$ $=(g, h):=g+i h$ called generator and a complex modulus $(l, p):=l+i p$ with real integer components $l$ and $p$. Alice and Bob independently select secret real integers alpha and beta respectively. Alice and Bob respectively compute their public keys:

$$
E:=L^{\text {alpha }} \bmod (l, p) \text {; }
$$

and

$$
F:=L^{\text {beta }} \bmod (l, p) .
$$

Then Alice and Bob compute respectively

$$
H_{1}:=F^{\text {alpha }} \bmod (l, p)
$$

and

$$
H_{2}:=E^{\text {beta }} \bmod (l, p) \text {. }
$$

As a result,

$$
M=H_{1}=H_{2}=\left(x_{0}, y_{0}\right):=x_{0}+i y_{0} .
$$

Therefore a pair of real integers $x_{0}$ and $y_{0}$ can be used by Alice and Bob to design a cryptographic protocol.

DHKE based on elliptic curves: Consider a modular elliptic curve (EC)

$$
y^{2}+e y=x^{3}+a x+b(\bmod p)
$$

where $a, b, e$ and $p$ are integer parameters of the EC, and modulus $p$ is an odd real prime [3]. If (6) is used for ECDHKE between Alice and Bob, then both parties create a mutual secret key $\left(x_{0}, y_{0}\right)$ that is a point on (6). The scheme is analogous to (1)-(5): Alice and Bob select a point $Q$ with high order on (6) and real integers alpha (Alice's secret key) and beta (Bob's secret key). Then they respectively compute their public keys:

$$
E:=\operatorname{alpha} \times Q \bmod p ;
$$

and

$$
F:=\operatorname{beta} \times Q \bmod p .
$$

Here both $E$ and $F$ are points on (6).

Then Alice and Bob compute respectively

$$
J_{1}:=\operatorname{alpha} \times F \bmod p ;
$$

and

$$
J_{2}:=\operatorname{beta} \times E \bmod p .
$$

As a result,

$$
M:=J_{1}=J_{2}:=\left(x_{0}, y_{0}\right) .
$$




\section{Decomposition}

Consider randomly selected non-zero integers $A \neq 1$; $B \neq 1 ; C \neq 1$ that are co-prime with $p$. Consider positive integers $k, q$ and $r$ that satisfy

$$
k+q+r=6 ; 1 \leq k, q, r \leq 4 .
$$

Compute $R:=y_{0}^{k} A \bmod p$;

$$
S:=x_{0}^{q} B \bmod p ; T:=y_{0}^{r} C \bmod p
$$

or $R:=x_{0}^{k_{i}} A ; S:=y_{0}^{q_{i}} B ; T:=x_{0}^{3-k_{i}-q_{i}} C(\bmod p)$;

\{for details see Step5 below\};

Select integers $u, v$ and $w$ that satisfy

$$
u+v+w=R ; \quad u+v-w=S ; \quad u-v+w=T .
$$

Then (14) implies that

$$
\begin{gathered}
u=(S+T)(p+1) / 2 \bmod p \\
v=(R-T)(p+1) / 2 \bmod p \\
w=(R-u-v) \bmod p .
\end{gathered}
$$

Here $k$ and $q$ are secret keys that satisfy

$$
1 \leq k \leq 4 ; 1 \leq q \leq 5-k ; r=6-k-q ;
$$

where $k$ and $q$ are periodically updated.

There are ten combinations of positive integers satisfying (12); these combinations are listed in lexicographically increasing order in Table $\mathbf{1 .}$

\section{Numeric Illustration}

Let $p=99991$; consider an elliptic curve

$$
y^{2}+1001 y=x^{3}+217(\bmod 99991) .
$$

Suppose Alice and Bob have established a secret key for communication as point $M=\left(x_{0}, y_{0}\right)=$ (86275, 81549); it is easy to verify that $P$ indeed satisfies (19). Juxtapose $\left(x_{0}, y_{0}\right)$ and let $G:=8627581549$.

\section{Information Hiding Protocol- $\{k, q\}$}

Step1: Communicating parties (Alice and Bob) establish a key $M=\left(x_{0}, y_{0}\right)$ using one of schemes listed in section 1;

Table 1. All combinations of exponents.

\begin{tabular}{ccccccccccc}
\hline States & 0 & 1 & 2 & 3 & 4 & 5 & 6 & 7 & 8 & 9 \\
\hline$k$ & 1 & 1 & 1 & 1 & 2 & 2 & 2 & 3 & 3 & 4 \\
$q$ & 1 & 2 & 3 & 4 & 1 & 2 & 3 & 1 & 2 & 1 \\
$r$ & 4 & 3 & 2 & 1 & 3 & 2 & 1 & 2 & 1 & 1 \\
\hline
\end{tabular}

Step2: Juxtapose coordinates $\left(x_{0}, y_{0}\right)$; let

$$
G=d_{1} d_{2} \cdots d_{t-1} d_{t},
$$

where $d_{i}$ are its decimal digits.

Here

$$
t \leq 2 \times\left\lceil\log _{10} p\right\rceil ;
$$

Step3: Suppose Alice wants to transmit a plaintext array

$$
m=\left\{m_{1}^{(1)}, \cdots, m_{5}^{(1)} ; \cdots ; m_{1}^{(i)}, \cdots, m_{5}^{(i)} ; \cdots ; m_{1}^{(s)}, \cdots, m_{5}^{(s)}\right\}
$$

where

$$
s=\lceil t / 5\rceil .
$$

Encryption of $m^{(i)}=\left\{m_{1}^{(i)}, m_{2}^{(i)}, \cdots, m_{5}^{(i)}\right\}$ :

Step4: Using $d_{i}$ select corresponding $\left\{k_{i}, q_{i}, r_{i}\right\}$ from Table 1;

Step5: if $d_{i}$ is even, then compute $R:=x_{0}^{k_{i}} A$; $S:=y_{0}^{q_{i}} B ; T:=x_{0}^{6-k_{i}-q_{i}} C(\bmod p)$;

else

$$
\begin{gathered}
R:=y_{0}^{k_{i}} A ; \\
S:=x_{0}^{q_{i}} B ; T:=y_{0}^{6-k_{i}-q_{i}} C(\bmod p) ;
\end{gathered}
$$

Step6: Compute the information hiding keys (15)-(17): $\{u, v, w\}$;

Step7 enhancement of crypto-immunity\}:

for $z \in\left\{u, v, w, x_{0}, y_{0}\right\}$ do

if $z<\sqrt{p}$, then $z:=p-2 z$ else $z:=2 z$;

Step8: compute

$$
\begin{gathered}
c_{1}^{(i)}:=m_{1}^{(i)} u ; c_{2}^{(i)}:=m_{2}^{(i)} v ; c_{3}^{(i)}:=m_{3}^{(i)} w(\bmod p) ; \\
c_{4}^{(i)}:=m_{4}^{(i)} x_{0} ; c_{5}^{(i)}:=m_{5}^{(i)} y_{0}(\bmod p) .
\end{gathered}
$$

Decryption is performed in reverse.

Remark2: After $t$ cycles Alice and Bob must use a DHKE to establish a new mutual secret key $G$.

Choice of $\boldsymbol{A}, \boldsymbol{B}$ and $\boldsymbol{C}$ : one way to choose $A$ and $B$ is to assign even digits of $G$ to $A$ and odd digits of it to $B$. Then select $C$ that is a multiplicative inverse of $A B$ modulo $p$ :

$$
C:=(A B)^{-1} \bmod p[4] .
$$

Remark3: The ideas of decomposition can be applied to any secret key; where splitting is completely independent of how this key is established.

\section{Plaintext Pre-conditioning}

If there is a pair $\left\{c_{j}^{i}, c_{l}^{i}\right\}$, where both components are 
smaller than $p$, then with high probability holds that $\operatorname{gcd}\left(c_{j}^{i}, c_{l}^{i}\right)>1$. Therefore either

$$
\operatorname{gcd}\left(c_{j}^{i}, c_{l}^{i}\right)=x_{0} \text { or } \operatorname{gcd}\left(c_{j}^{i}, c_{l}^{i}\right)=y_{0} .
$$

To preclude this possibility consider the following protocol of plaintext pre-conditioning: subdivide plaintext $m$ into arrays of blocks in such a way that for every block $m_{r}$ holds $m_{r} \leq(p-2) / 2$; if $m_{r} \leq \sqrt{p}$, then assign

$$
m_{r}:=p-2 m_{r}
$$

Remark4: Notice that the right-most binary digit of $m_{r}$ equals 1 .

\section{Numeric Illustration Continued}

Assign all even digits of $G:=8627581549$ to $A$ and all odd digits of $G$ to $B$.

Then $A=67859$, in Table 2 they are shown in bold font $\}, B=82514$, and $C=(A B)^{-1} \bmod p=87964$ [3].

Indeed: $(21508 * 87964) \bmod 99991=1$.

Since $G$ does not have digits 0 and 3, then only eight of ten combinations of $\{k, q, r\}$ that are listed in Table 1 are used to compute the information hiders $u, v, w$ :

Computation of encryptors $u, v, w$

For $d_{1}=8$ see the $1^{\text {st }}$ column in Table 2 \} compute

$$
\begin{aligned}
& R:=x_{0}^{k_{i}} A=86275^{3} \times 67859 \bmod p ; \\
& S:=y_{0}^{q_{i}} B=81549^{2} \times 82514 \bmod p ; \\
& T:=x_{0}^{3-k_{i}-q_{i}} C=x_{0}^{1} C \bmod p ;
\end{aligned}
$$

and then compute encryptors $u, v$ and $w$ (15)-(17).

For $d_{2}=6 \quad$ see the $2^{\text {nd }}$ column in Table 2 \} compute

$$
\begin{aligned}
& R:=x_{0}^{k_{i}} A=86275^{2} \times 67859 \bmod p ; \\
& S:=y_{0}^{q_{i}} B=81549^{3} \times 82514 \bmod p ; \\
& T:=x_{0}^{3-k_{i}-q_{i}} C=x_{0}^{1} \times 87964 \bmod p ;
\end{aligned}
$$

and then compute encryptors $u, v$ and $w$.

See Table 3 of all encryptors for $i=1,2, \cdots t$.

\section{Plaintext pre-conditioning}

$$
\text { Let } \begin{aligned}
m^{(1)} & =\left\{m_{1}^{(1)}, m_{2}^{(1)}, m_{3}^{(1)}, m_{4}^{(1)}, m_{5}^{(1)}\right\} \\
& =\{266,45769,37585,36488,46572\} .
\end{aligned}
$$

Remark5: Notice that each component in $m$ is smaller

Table 2. Sequence of exponents $k, q$ and $\boldsymbol{r}$ based on secret key $\left(x_{0}, y_{0}\right)$.

\begin{tabular}{ccccccccccc}
\hline States & 8 & $\mathbf{6}$ & 2 & $\mathbf{7}$ & 5 & $\mathbf{8}$ & 1 & $\mathbf{5}$ & 4 & $\mathbf{9}$ \\
\hline$k$ & 3 & 2 & 1 & 3 & 2 & 3 & 1 & 2 & 2 & 4 \\
$q$ & 2 & 3 & 3 & 1 & 2 & 2 & 2 & 2 & 1 & 1 \\
$r$ & 1 & 1 & 2 & 2 & 2 & 1 & 3 & 2 & 3 & 1 \\
\hline
\end{tabular}

Table 3. Encryption stage: information hiders $u, v, w$ and ciphertexts.

\begin{tabular}{ccccc}
\hline$d_{i}$ & $d_{1}=8$ & $d_{2}=6$ & $\cdots$ & $d_{t}=9$ \\
\hline$R$ & 02480 & 30939 & $\cdots$ & 21751 \\
$S$ & 86137 & 18463 & $\ldots$ & 36105 \\
$T$ & 77173 & 77173 & $\ldots$ & 21896 \\
$u$ & 81655 & 47818 & $\ldots$ & 78996 \\
$v$ & 12649 & 15594 & $\ldots$ & 49923 \\
$w$ & 08167 & 67518 & $\cdots$ & 92814 \\
\hline
\end{tabular}

than $(p-1) / 2$.

Because $m_{1}<\sqrt{p}$, reassign $m_{1}:=p-2 m_{1}$; \{odd integer .

Since all other blocks in plaintext $m$ are greater than $\sqrt{p}$, therefore reassign

$$
\begin{aligned}
& m_{2}:=2 m_{2} ; m_{3}:=2 m_{3} ; \\
& m_{4}:=2 m_{4} ; m_{5}:=2 m_{5}
\end{aligned}
$$

\{all four are even integers $\}$.

Encryption $\{$ see Step7\}:

$c_{1}:=m_{1} u \bmod p ; c_{2}:=m_{2} v \bmod p$;

$c_{3}:=m_{3} w \bmod p$;

$c_{4}:=m_{4} x_{0} \bmod p ; c_{5}:=m_{5} y_{0} \bmod p$.

Alice sends ciphertext $\left\{c_{1}, . ., c_{5}\right\}$ to Bob via open communication channels. See Table 3 with encryptors $R$, $S, T, u, v$ and $w$; Table 4 with plaintext arrays $m_{j}^{(i)}$ and Table 5 of corresponding ciphertext arrays.

Table 4. Plaintext arrays $m_{j}^{(i)} ; i=1,2, \cdots, t$.

\begin{tabular}{ccccc}
\hline $\boldsymbol{i}$ & 1 & 2 & $\cdots$ & $t$ \\
\hline$m_{1}^{(i)}$ & 00266 & 08764 & $\cdots$ & 38643 \\
$m_{2}^{(i)}$ & 45769 & 43654 & $\cdots$ & 00179 \\
$m_{3}^{(i)}$ & 37585 & 34631 & $\cdots$ & 07320 \\
$m_{4}^{(i)}$ & 36488 & 45731 & $\cdots$ & 34219 \\
$m_{5}^{(i)}$ & 46572 & 00301 & $\cdots$ & 04352 \\
\hline
\end{tabular}

Table 5. Ciphertext arrays $c_{j}^{(i)} ; i=1,2, \cdots, t$.

\begin{tabular}{ccccc}
\hline$i$ & 1 & 2 & $\cdots$ & $t$ \\
\hline$c_{1}^{(i)}$ & 70985 & 29342 & $\ldots$ & 34378 \\
$c_{2}^{(i)}$ & 68373 & 03496 & $\ldots$ & 25955 \\
$c_{3}^{(i)}$ & 68641 & 52628 & $\ldots$ & 19261 \\
$c_{4}^{(i)}$ & 71085 & 94285 & $\ldots$ & 19900 \\
$c_{5}^{(i)}$ & 83732 & 03083 & $\ldots$ & 66378 \\
\hline
\end{tabular}


Decryption is performed in reverse: since Bob knows the mutual secret key $M=\left(x_{0}, y_{0}\right)$, he finds $A, B, k, q$ and $r$; then computes $C, R, S, T$; and the multiplicative inverse values of $u, v$ and $w$.

\section{Key Establishment Based on Gaussian Modulus}

Consider $(l, p)=(1000,3001)$; and a generator $L=(2269$, -2204). All corresponding steps and actions by Alice and Bob are provided in Table 6.

Therefore, $M=(-0502,1853)$ is the mutual secret key established between Alice and Bob. Notice that components in $M$ can be positive and negative. If a component is negative, post digit " 2 " in front of its left-most digit; if the component is positive, post digit " 9 " in front of its left-most digit. Therefore $M:=(20502,91853)$. For large $l$ and $p$ in modulus $(l, p)$, the probability is negligibly small that either $x_{0}=0$ or $y_{0}=0$.

\section{Computational Complexity}

For every digit in juxtaposed $G$ it is possible to encrypt one plaintext array.

With high probability each component in $\left(x_{0}, y_{0}\right)$ has the same number of digits $t$ as modulus $p$. Therefore in $G$ there are about $2 t$ digits. For each digit we select an appropriate combination of keys $\{k, q, r\}$ from Table 1 and encrypt five blocks of the plaintext. Therefore for every $G$ we can encrypt $N(p)=5 \times 2 t=10 t=10\left\lceil\log _{10} p\right\rceil$ blocks.

In application, to assure strong crypto-immunity, $t=2\left\lceil\log _{10} p\right\rceil \in[100,200]$.

Thus, if $p$ is a 50-digit long integer, then $N(p)=10 \times 50=500$ blocks of plaintext.

\section{Reduction of Complexity}

To reduce computational complexity of encryption for every $G$, we pre-compute and store for every $f=1, \cdots, 4$ $D x_{0}^{f} \bmod p$ and $D y_{0}^{f} \bmod p$, where $D \in\{A, B, C\}$.

Another way to reduce complexity is to avoid computation of multiplicative inverse $C$ (24). Instead we can partition $G$ onto about equal number of digits. For instance, if $G=\underline{27182818284} \underline{59045}$, we can either assign $A_{1}:=$ 27182; $B_{1}:=\mathbf{8 1 8 2 8 4}$ and $C_{1}:=59045$ or to substitute di-
Table 6. Key establishment (1)-(5).

\begin{tabular}{lll}
\hline Keys & Alice's action & Bob’s action \\
\hline Secret & Alpha $=1913$ & Beta $=1999$ \\
Public & $E=(-846,1022)$ & $F=(439,2876)$ \\
Private & $H_{1}=F^{\text {alpha }}$ & $\begin{array}{r}H_{2}=E^{\text {beta }} \\
\text { key }\end{array}$ \\
& $=(-0502,1853)$ & $=(-0502,1823)$ \\
\hline
\end{tabular}

gits $1,2, \cdots, 9,1,2, \cdots$ into $G$ :

$$
\begin{aligned}
& A_{2}:=1728384858657085 ; \\
& B_{2}:=2112231425469748 ; \\
& C_{2}:=2718283848556075 .
\end{aligned}
$$

\section{Conclusion}

In the proposed cryptocol it is shown that for every pair of integers in secret key $\left(x_{0}, y_{0}\right)$ there are numerous ways to compute integers $\{u, v, w\}$ that hide information on the encryption stage.

\section{Acknowledgements}

I express my appreciation to NJIT students A. Koripella and M. Sikorski for their assistance and suggestions that improved style of this paper, to D. Kanevsky for his participation in discussion, and to C. Pomerance and $\mathrm{H}$. Cohen for their advices about complex modulus reduction.

\section{References}

[1] W. Diffie and M. Hellman, "New Directions in Cryptography," IEEE Transactions on Information Theory, Vol. IT-22, No. 6, 1976, pp. 644-654. doi:10.1109/TIT.1976. 1055638

[2] S. G. Krantz, "Modulus of a Complex Number," Handbook of Complex Variables, Birkhäuser Publishing Ltd., Boston, 1999, pp. 2-3.

[3] J. Hoffstein, J. Pipher and J. Silverman, “An Introduction to Mathematical Cryptography,” Springer, New York, 2008.

[4] B. Verkhovsky, "Enhanced Euclid Algorithm for Modular Multiplicative Inverse and Its Application in Cryptographic Protocols," International Journal of Communications, Network and System Sciences, Vol. 3, No.12, 2010, pp. 901-906. doi:10.4236/ijcns.2010.312123 


\section{Appendix}

\section{A1. Generalization}

Step1A: Establish a secret key $M$ between communicating parties and juxtapose it.

Remark6: Either Gaussian arithmetic with complex modulus or other mechanisms for DHKE can be used to establish $M$.

Step2A: Using $M$, the parties select $A_{1}, \cdots, A_{s}$, where $s$ is an integer parameter of encryption protocol;

Step3A: for $i=1, \cdots, t$ for $j=1, \cdots, s$ do

$$
\begin{aligned}
& \text { if } \quad d_{i}=j(\bmod 2) \\
& \text { then } R_{j}:=A_{j} x_{0}^{k_{j}} \bmod p, \\
& \text { else } R_{j}:=A_{j} y_{0}^{k_{j}} \bmod p ;
\end{aligned}
$$

Step4A: Compute for $j=2,3, \cdots, s$

and

$$
u_{j}:=\left[\left(R_{1}-R_{j}\right) / 2\right] \bmod p ;
$$

$$
u_{1}=\left[R_{1}-\left(u_{2}+. .+u_{s}\right)\right] \bmod p ;
$$

$$
c_{i}:=m_{i} u_{i} \bmod p .
$$

A2. Selection of Table for $k_{1}, k_{2}, \cdots k_{s}$

If $s>3$, the number of possible combinations for secret keys $k_{1}, k_{2}, \cdots k_{s}$ grows exponentially if $s$ is increasing.

This is an additional potential for randomization. If a protocol designer of encryption/decryption scheme represents $G$ in a numeric form with base $n$, then it is possible to select $n$ combinations of secret exponents $k_{1}, k_{2}, \cdots k_{s}$, where each combination corresponds to every digit of $G$. Therefore the parties must exchange between themselves a $n \times n$ square matrix:

$$
\left(\begin{array}{ccc}
k_{11} & \ldots & k_{1 n} \\
\vdots & \ddots & \vdots \\
k_{n 1} & \cdots & k_{n n}
\end{array}\right)
$$

For example, if $s=4$ and $n=16$, then we need to specify sixteen combinations of $k_{1}, k_{2}, . . k_{s}$ and $k_{4}$.

If $1 \leq k_{i} \leq 5$ and $k_{1}+\cdots+k_{4}=d$, then the number of possible combinations of $k$ 's is 35 for $d=8$.

Step5A \{encryption\}: for $i$ from 1 to $s$ 